\title{
Survivin-T34A: molecular mechanism and therapeutic potential
}

\section{Jonathan R Aspe}

Nathan R Wall

Center for Health Disparities Research and Molecular Medicine, Division of Biochemistry and Microbiology, Department of Basic Sciences, Loma Linda University, Loma Linda, CA, USA
Correspondence: Nathan R Wall Center for Health Disparities Research and Molecular Medicine, Division of Biochemistry and Microbiology, Department of Basic Sciences, Loma Linda University, I 1085 Campus Street, Mortensen Hall, Room I62, Loma Linda, CA 92350, USA

Tel +l 9095584000 Ext 8I397

$\mathrm{Fax}+19095580177$

Email nwall@llu.edu
This article was published in the following Dove Press journal:

OncoTargets and Therapy

3 December 2010

Number of times this article has been viewed
Abstract: The inhibitor of apoptosis protein survivin's threonine 34 to alanine (T34A) mutation abolishes a phosphorylation site for p34(cdc2)-cyclin B1, resulting in initiation of the mitochondrial apoptotic pathway in cancer cells; however, it has little known direct effects on normal cells. The possibility that targeting survivin in this way may provide a novel approach for selective cancer gene therapy has yet to be fully evaluated. Although a flurry of work was undertaken in the late 1990s and early 2000s, only minor advances on this mutant have recently taken place. We recently described that cells generated to express a stable form of the mutant protein released this survivin-T34A to the conditioned medium. When this conditioned medium was collected and deposited on naive tumor cells, conditioned medium T34A was as effective as some chemotherapeutics in the induction of tumor cell apoptosis, and when combined with other forms of genotoxic stressors potentiated their killing effects. We hope with this review to revitalize the T34A field, as there is still much that needs to be investigated. In addition to determining the therapeutic dose and the duration of drug therapy required at the disease site, a better understanding of other key factors is also important. These include knowledge of target cell populations, cellsurface receptors, changes that occur in the target tissue at the molecular and cellular level with progression of the disease, and the mechanism and site of therapeutic action.

Keywords: survivin, T34A, apoptosis, proliferation, therapy

\section{Survivin is important in cancer}

Apoptosis is a physiological cell suicide program that is critical for the development and maintenance of healthy tissues. ${ }^{1}$ The evasion of apoptosis, as identified by Hanahan and Weinberg, is a primary characteristic of human cancer ${ }^{2}$ with proteins involved in its control of significant biological interest, as they could prove to be important targets for therapy development. The inhibitor of apoptosis (IAP) family consists of proteins with a proven ability to inhibit apoptosis, although much of how this works mechanistically is still to be revealed. Survivin is an important IAP because its expression is among the most tumor specific of all human gene products. ${ }^{3,4}$ It has been classified as a bifunctional protein, as it can inhibit apoptosis and cell cycle. ${ }^{5}$ More specifically, survivin's promoter exhibits M-phase-inducible transactivation and, in HeLa cells, both survivin protein and mRNA are upregulated at the $\mathrm{G}_{2} / \mathrm{M}$-phase of the cell cycle. ${ }^{5}$ Survivin expression is enhanced during embryonic and fetal development but not in terminally differentiated normal tissue. ${ }^{6}$ Its expression has also been seen in virtually every type of human cancer, ${ }^{7}$ making survivin an attractive protein in the study of cancer pathophysiology, drug discovery, and medical diagnosis. Our laboratory results have recently shown that cancer cells release survivin into the extracellular space ${ }^{8}$ 
packaged in small 50-150 nm vesicles called exosomes. ${ }^{9}$ This is significant, as it may reveal new mechanisms about how cancer cells communicate with one another and how they affect the tumor microenvironment. We hope that this discovery may provide other options for targeted therapy. However, this discovery begs for studies about why and how survivin is packaged within exosomes, how survivin from exosomes is utilized by the recipient cells, and what are the effects of exosomal survivin on normal human cells.

Survivin physically associates with and is phosphorylated on its threonine $34\left(\mathrm{Thr}^{34}\right)$ by the cyclin-dependent kinase p34(cdc2)-cyclin B1 protein complex. ${ }^{10}$ Loss of $\mathrm{Thr}^{34}$ phosphorylation results in dissociation of the caspase-9-survivin protein complex and caspase-dependent apoptosis. ${ }^{10-12}$ Initially, $\mathrm{Thr}^{34}$ through site-directed mutagenesis was changed to Ala (T34A) as a nonphosphorylated mimic of survivin. ${ }^{10}$ When replication-deficient adenoviruses encoding this survivin-T34A mutant were produced and used to infect cancer cell lines, spontaneous apoptosis resulted. In contrast, the viability of normal human cells, including fibroblasts, endothelium, and smooth muscle cells, was unaffected. ${ }^{11}$ In studies analyzing normal human oral mucosa, oral submucosa fibrosis, and oral squamous cell carcinomas, ${ }^{13}$ a gradation from no survivin $\mathrm{Thr}^{34}$ phosphorylation in normal to significantly enhanced amounts of phosphorylated survivin $\mathrm{Thr}^{34}$ in the cancer tissues was recorded. Furthermore, phosphorylated $\mathrm{Thr}^{34}$ and $\mathrm{p} 34(\mathrm{cdc} 2)$ kinase expression was not detected in normal tissue samples but increased with advancing stages in the cancer tissue samples. ${ }^{13}$ Agents that elevate $\mathrm{p} 34(\mathrm{cdc} 2)$ kinase activity during spindle checkpoint activation drive increased survivin expression and cancer cell viability ${ }_{1}^{14}$ resulting in limited anticancer effects. Pharmacologic, genetic, or molecular ablation of p34(cdc2) kinase after microtubule stabilization resulted in apoptosis independent of p53, suppression of tumor growth, and indefinite survival without toxicity in mice. ${ }^{14}$ Taken together, the ablation of survivin-dependent apoptosis inhibition could improve the efficacy of many agents used to treat cancer.

One of the most daunting questions in any gene-based approach for cancer treatment is in the method of delivery. We have recently shown that survivin exists in a novel extracellular pool in tumor cells. Furthermore, we have constructed stable cell lines that provide the extracellular pool with either wildtype survivin or dominant-negative mutant survivin (T34A). ${ }^{8}$ Cancer cells grown in the conditioned medium taken from wild-type survivin-expressing cells absorbed survivin and experienced enhanced protection against genotoxic stress as well as exhibited an increased replicative and invasive potential. ${ }^{8}$ Alternatively, cancer cells grown in conditioned medium from T34A cells began to apoptose through a caspase-2- and caspase-9-dependent pathway that was further enhanced by the addition of chemo- and radiotherapeutic modalities. ${ }^{8}$ We believe that this extracellular survivin-T34A could be used as a possible 'white knight' to survivin's 'dark family'. The following sections detail T34A's journey as a novel potential cancer therapeutic. The aforementioned studies have already provided impetus for further studies on T34A, but much is still unknown. The advancement of future T34A studies may provide a more comprehensive understanding of cancer and the potential of using T34A to battle it.

\section{T34A induces apoptosis}

What makes this survivin-T34A mutant important as a potential therapeutic is that little or no toxicity has resulted from its treatment in vitro or in vivo. ${ }^{12,15,16} \mathrm{~T} 34 \mathrm{~A}$-treated mice showed significantly longer survival rates compared with control mice as well as having no pathologic changes in the major organs. ${ }^{17}$ More specifically, tumor cells treated in vitro with T34A exhibited reduced cell survival that was associated with morphological features of apoptosis. ${ }^{8,11,15,16,18-28}$ Studies of mouse xenographs treated with survivin-T34A concluded with significant tumor size reduction and increased animal survival rates ${ }^{15-17,19,27}$ except in mice subcutaneously injected with the IGROV1 ovarian carcinoma cell line, where there were no significant differences between T34A-infected tumors and controls. ${ }^{18}$ These results may be explained, however, as IGROV1 cells do not express significant levels of endogenous survivin and T34A, as shown in normal survivin-nonexpressing cells, does not induce apoptosis. ${ }^{11}$ It was not until IGROV1 cells were infected with adenovirus vector-expressing caspase-3 (Ad-caspase-3) or coinfected with Ad-caspase-3 and Ad-T34A that tumor size was reduced and survival rates extended. ${ }^{18}$ It is of much interest that the adenoviruses used in these studies upregulated survivin mRNA, as shown by reverse transcription-polymerase chain reaction, and concomitantly resulted in a half-fold reduction in another IAP, XIAP (X-linked inhibitor of apoptosis protein).$^{18}$ Furthermore, in melanoma cells that have been engineered to express an inducible survivin-T34A, ${ }^{29}$ XIAP and the IAP Livin were cleaved upon T34A induction. ${ }^{20}$ These reductions may in part be responsible for the more favorable outcomes observed in these studies. In contrast, it has been postulated that the transfection of cells may affect endogenous levels of XIAP, but there was no effect on XIAP protein levels following transfections using virus vector controls or Ad-survivin or Ad-T34A treatment. ${ }^{11,18,20}$ There were also 
no changes in other IAPs or members of the Bcl-2 family. ${ }^{20}$ Although survivin-T34A may not affect endogenous levels of other IAPs, a survivin-IAP (XIAP, cIAP1, and cIAP2) complex has been confirmed, as well as a suggestion for direct interaction between survivin and XIAP through their BIR (baculovirus IAP repeat) domains. ${ }^{30}$ Furthermore, evidence that T34A can only kill cells expressing survivin has been shown in its inability to kill normal human cells treated in vitro ${ }^{11,31}$ and in vivo. ${ }^{11,15}$ However, T34A has shown significant killing effects in normal endothelial cells that express high levels of endogenous survivin. ${ }^{19}$ Whether this is the result of some interaction between endogenous survivin, other IAPs and the T34A mutant, or a competition between them for protein interactors is currently unknown.

Mechanistic examination of T34A-induced apoptosis revealed mitochondrial events that preceded caspase activation, including depolarization and the release of cytochrome c, AIF (apoptosis-inducing factor), and Smac/ DIABLO (second mitochondria-derived activator of caspase/direct inhibitor of apoptosis-binding protein with low isoelectric point). ${ }^{11,19,20,32}$ Also, T34A treatment of melanoma cells has been shown to involve Apaf-1, Mcl-1, and PARP (poly [ADP-ribose] polymerase) cleavage. ${ }^{19,20,25}$ Stress-induced cytochrome c release results in apoptosome formation and caspase activation; ${ }^{33}$ thus, several groups have reported that caspase-3 may be important to T34A-induced apoptosis. ${ }^{11,18-21,26,34}$ A significant increase in apoptosis was reported in ovarian carcinoma cells treated with T34A and caspase- 3 in vivo and in vitro. ${ }^{18} \mathrm{We}$, however, when working with cervical carcinoma HeLa cells, were unable to show cleavage of caspase- 3 or caspase- 8 , though cleavage of caspase- 9 and caspase- 2 was recorded. ${ }^{8}$ Further experiments revealed that $\mathrm{T} 34 \mathrm{~A}$ induced a reduction in the proforms of caspase-2, caspase-7, and caspase-9; a cleavage of PARP; and an induction of mitochondrial depolarization. Treatment with caspase inhibitors Z-DEVD-FMK and Z-VAD-FMK has been shown to counter the effect of T34A treatment, suggesting that T34A-induced apoptosis may act through the caspase-dependent pathway. ${ }^{20}$ Additionally, the earliest proapoptotic event observed in T34A-treated cells was nuclear translocation of mitochondrial AIF, which triggers both mitochondrial-associated apoptosis and caspase-independent DNA fragmentation..$^{20}$ Taken together, survivin-T34A targets the intrinsic ${ }^{35}$ or mitochondria-associated apoptotic pathway to most probably induce caspase-dependent and independent apoptosis (Figure 1). However, with the exception of one study, ${ }^{36}$ survivin has not been shown to directly inhibit caspases, and whether survivin-T34A disrupts caspase activity has yet to be fully elucidated. We postulate that survivin-T34A-induced apoptosis begins further upstream in the intrinsic apoptotic pathway initiating mitochondrial depolarization as well as inhibition of IAPs known to directly inhibit caspases (eg, XIAP-caspase-3).

The tumor suppressor gene, p53, has been widely studied and is known to induce apoptosis. ${ }^{37,38}$ As a transcription factor, p53 is activated by a variety of genotoxic and cytotoxic stresses. Upon activation, p53 prevents the proliferation of genetically compromised cells by regulating the expression of a battery of genes that initiate cell cycle arrest, apoptosis, and DNA repair (Figure 2). ${ }^{39}$ Transcriptionally, p53 activates Mdm2, which in turn targets p53 for ubiquitin-proteasomedependent degradation. In response to stress, a decrease in Mdm2 protein levels and/or its activity and the interaction between Mdm2 and p53 lead to p53 stabilization. ${ }^{40}$ The relationship between survivin and p53 may be important, as survivin regulates cell division and inhibits apoptosis, whereas p53 inhibits cell cycle progression and induces apoptosis. ${ }^{21}$ Survivin was recently shown to be transcriptionally repressed by wild-type p53, and when overexpressed in cells sensitive to p53-dependent cell death markedly inhibited apoptosis induced by ultraviolet light. ${ }^{41}$

When cancer cells that had been stably transfected with survivin-T34A were treated with the DNA damage-inducing Adriamycin, p53 protein levels were significantly elevated compared with DNA damage alone or when combined with stably transfected survivin. ${ }^{21}$ Increased levels of p53 mRNA preceded the elevation in p53 protein levels seen with T34Atreated cells, suggesting that p53 may be directly upregulated by survivin-T34A. Survivin disruption by survivin-T34A also resulted in the degradation of $\mathrm{Mdm} 2$. Proteasome inhibition and caspase-3 inhibition blocked this survivin-T34Aassociated decrease in $\mathrm{Mdm} 2$, resulting in the stabilization of p53. Survivin-T34A was not shown to affect mRNA levels of Mdm2. ${ }^{21}$ Furthermore, the p53 homologs TAp63, $\Delta \mathrm{Np} 63$, TAp73, and $\Delta \mathrm{Np} 73$ were analyzed for changes following survivin-T34A treatment. The response of these $\mathrm{p} 53$ homologs is important because TAp63 and TAp73 both transactivate similar genes as $\mathrm{p} 53$, whereas the $\Delta \mathrm{Np} 63$ and $\Delta \mathrm{Np} 73$ function as antagonists to p53 and the TA homologues. ${ }^{42-44}$ $\Delta \mathrm{Np} 63$, TAp73, and $\Delta \mathrm{Np} 73$ were all decreased in response to survivin-T34A, whereas TAp63 was not detectable. These findings suggest that $\mathrm{p} 53$ transcription may be differentially regulated by survivin-T34A. ${ }^{21}$ Taken together, the ability of survivin-T34A to perturb the p53/Mdm2 feedback loop, resulting in increasing levels of p53 and p53's ability to then transcriptionally repress survivin, may prove to be just 


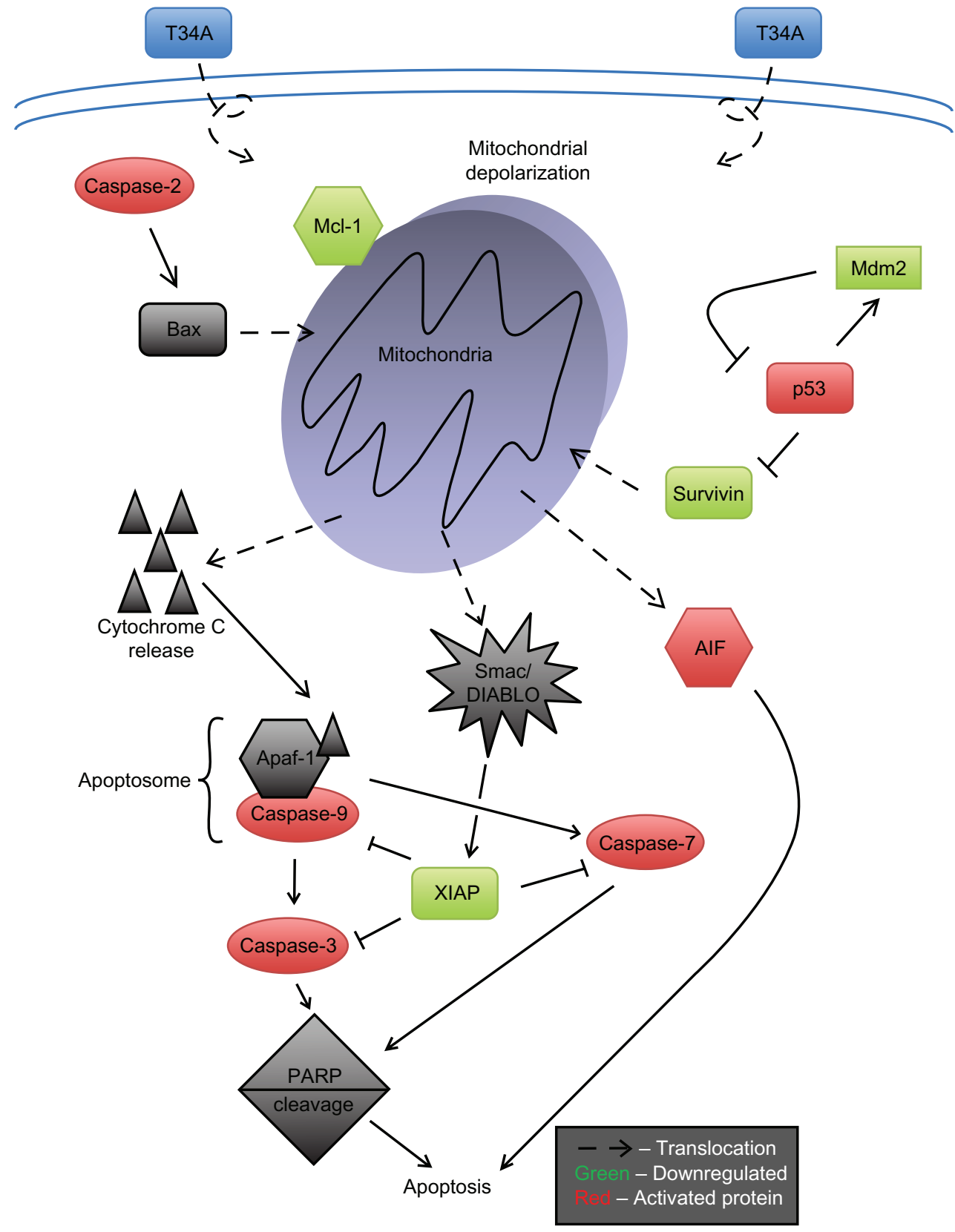

Figure I Survivin-T34A induces apoptosis by modulating the intrinsic (mitochondrial) pathway of apoptosis. After the mitochondrial depolarization, mitochondrial proteins that activate caspases, such as cytochrome $\mathrm{c}$ and Smac/Diablo, as well as those that are caspase independent, such as AlF, are released by way of transmembrane channels across the mitochondrial outer membrane.

the therapeutic strategy needed for targeting p53-associated stress-induced repair pathways in cancer.

\section{Survivin $\mathrm{Thr}^{34}$ plays a role in cell cycle regulation}

Survivin is a structurally unique IAP family protein that is expressed in mitosis in a cell cycle-dependent fashion and localized to components of the mitotic apparatus. ${ }^{5}$ Survivin has been described as bifunctional, in that it is involved in both inhibition of apoptosis and control of cell division. ${ }^{45,46}$
In order to study the effect survivin phosphorylation at $\mathrm{Thr}^{34}$ had on cell division, the nonphosphorylatable T34A form of survivin was compared with T34E, which acts as a phosphomimetic. ${ }^{34}$ As a phosphomimetic, T34E assumes the role of a phosphorylated $\mathrm{Thr}^{34}$ and therefore mimics an activated survivin. T34A-treated cells grew two-fold faster than control cells, whereas T34E cells grew 2.5 -fold slower. Depletion studies showed that only T34A was normally distributed and able to support cell proliferation, whereas T34E was not. 


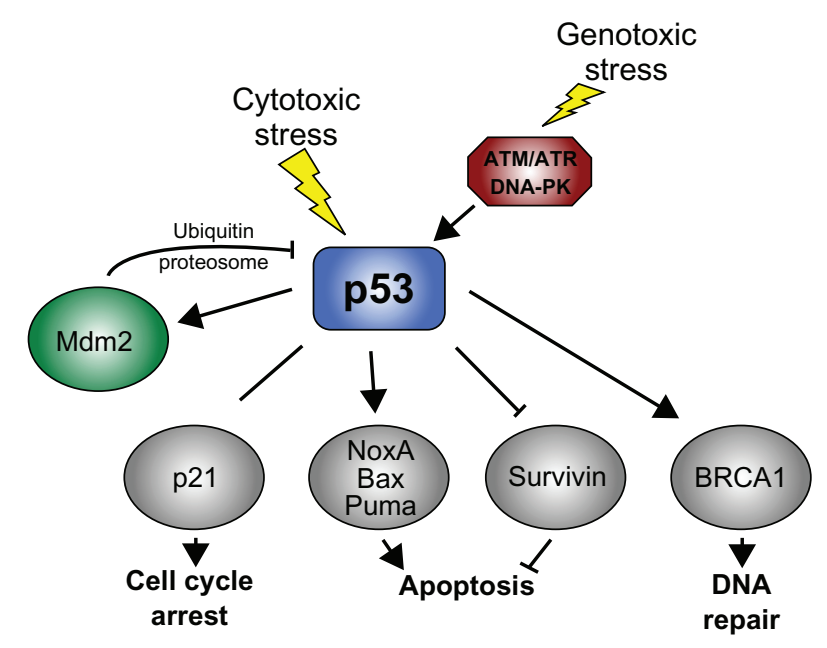

Figure 2 Survivin-T34A promotes p53-associated apoptosis. Upon activation, p53 prevents the proliferation of genetically compromised cells by regulating the expression of a battery of genes that initiate cell cycle arrest, apoptosis, and DNA repair. Survivin disruption by T34A treatment of cancer cells resulted in increased p53 protein levels and proteasomal degradation of Mdm2.

Survivin has been known to be upregulated in $\mathrm{G} 2 / \mathrm{M}$ of the cell cycle, but T34A's ability to induce apoptosis is $\mathrm{G} 2 / \mathrm{M}$ independent. ${ }^{18}$ The cell cycle profile of T34A-treated cells was normal, whereas T34E-treated cells had a significantly increased $\mathrm{G} 2 / \mathrm{M}$ population, suggesting that cell division failed. ${ }^{34}$ Furthermore, cancer cells that exhibited endogenous levels of functional survivin when treated with T34A showed significant mitotic index inhibition, ${ }^{11}$ as well as a reduction in colony formation. ${ }^{8}$ Ultimately, when endogenous survivin was depleted, T34A restored survivin's mitotic function, whereas T34E was mitotically incompetent. We therefore conclude that phosphorylated $\mathrm{Thr}^{34}$ inhibits mitosis,${ }^{34}$ but nonphosphorylated survivin is important for mitosis.

T34A has been shown to decrease the proportion of cells in S-phase following S-phase arrest. ${ }^{26}$ Evidence for survivinT34A regulation of chromosomal passenger complexes and central spindle checkpoint during mitosis has been generated..$^{47}$ In this work, survivin-T34A was also shown to be able to restore the spindle checkpoint defect following mitosis arrest, unlike the other non-wild-type forms, as well as rescue the cells from cytokinesis failure. ${ }^{47}$ These findings strongly suggest that survivin-T34A is a functional complement for endogenous survivin. Because survivin-T34A functionally behaves as survivin, it can localize appropriately during mitosis and thus correctly colocalize Aurora B at the centromeres and midzone, ${ }^{34,47}$ allowing proper mitosis regulation. Further experiments show that survivin-T34A correctly localizes BubR1 to the kinetochores and can restore localization of endogenous Borealin/Dasra B to both centromeres and central spindles. ${ }^{48}$ Taken together, survivin-T34A allows for the enhanced proliferative effects of survivin, whereas survivin-T34E does not. This suggests that the nonphosphorylated survivin $\mathrm{Thr}^{34}$ (T34A) accounts for cellular proliferation as opposed to the phosphorylated survivin $\mathrm{Thr}^{34}$ (T34E). Also, contrasting survivin $\mathrm{Thr}^{34}$ for its cytoprotective activity revealed that $\mathrm{T} 34 \mathrm{E}$ is responsible for the antiapoptotic characteristics, whereas T34A allows for caspase activation and induced apoptosis. ${ }^{34}$

\section{T34A opposes classical cancer characteristics}

Angiogenesis is crucial for cell survival and function, and without it tumor cells are not able to obtain oxygen and nutrients. Targeting tumor angiogenesis is a relatively new therapeutic strategy, and it is believed that if the tumor's oxygen and nutrient supply is limited, tumor growth and development will be inhibited and may result in apoptosis. Survivin-T34A treatment of tumor-bearing mice resulted in inhibited tumor-induced angiogenesis and increased apoptosis. ${ }^{16,17,27,49}$ Tumor sections taken from human breast cancer transplants in mice were stained with anti-CD31 antibody to determine microvessel density. ${ }^{50}$ Survivin-T34Atreated groups showed significantly reduced microvessel density compared with controls. ${ }^{16}$ In vivo results showed T34A-induced apoptotic characteristics in murine endothelial cells as well as decreased new vessel formation when compared with control mice. ${ }^{16,19}$ Furthermore, experiments using CT26 colorectal cancer cells showed similar results. ${ }^{17}$ It is still unknown how survivin-T34A treatment reduces tissue staining for the angiogenesis marker CD31. The elucidation of this mechanism may provide insight into this tumor-acquired capability. Survivin has been shown to be upregulated by vascular endothelial growth factor (VEGF), ${ }^{19}$ which is an important promoter of angiogenesis. Perhaps survivin regulation by VEGF promotes angiogenesis by protecting developing vessels from apoptosis. Therefore, survivin-T34A inhibits VEGF's promotion of angiogenesis, which may be mediated through loss of $\mathrm{p} 53$, activation of phosphatidylinositol 3'-kinase, or phosphorylation of signal transducers and activators of transcription 3. ${ }^{51-55}$

Once a tumor mass becomes constricted, cells acquire the ability to invade surrounding tissue and metastasize to distant sites, which accounts for $90 \%$ of human cancer deaths. ${ }^{2}$ The inhibition of survivin using adenoviruses (Ad-T34A) or short hairpin RNA dramatically inhibited invasiveness of prostate cancer cells in the in vitro invasion assay and spontaneous metastasis in the Dunning prostate cancer in vivo model. ${ }^{56}$ Treatment of subcutaneous breast cancer tumors in mice 
using T34A plasmids ${ }^{16}$ or Ad-T34A ${ }^{11}$ inhibited metastasis of breast tumor cells from the primary tumor site to the lungs and peritoneum, respectively. ${ }^{16}$ Specifically, control treatment groups were observed to have a greater number of metastatic nodules and structural destruction of pulmonary alveoli compared with the T34A treatment group in transplanted mice. ${ }^{11,16}$ In light of the role that metastasis plays in cancer death, T34A's metastasis-inhibitory role is important, as current therapeutics offer limited capabilities.

Cancerous lesions promote tumor growth, motility, invasion, and angiogenesis via oncogene-driven immunosuppressive leukocyte infiltrates. ${ }^{57} \mathrm{~T} 34 \mathrm{~A}$ has been shown to enhance T-lymphocyte activity against autologous tumor cells. ${ }^{16}$ In addition, T-cells derived from mouse xenographs exhibited higher cytotoxicity against target tumor cells in T34A-treated mice. ${ }^{17}$ Further studies revealed that adoptive transfer of $\mathrm{CD}^{+}$T-lymphocytes, and not their $\mathrm{CD}^{+} \mathrm{T}$-lymphocyte counterparts, isolated from the spleen of T34A-treated mice exhibited a decrease in tumor volume. Perhaps the treatment of tumor cells with T34A or with immune cells that have been primed by survivin-T34A association will redress the immunosuppression associated with cancer.

\section{Translational approaches and obstacles}

Given survivin's role in chemotherapy and radiation resistance, ${ }^{11,12,22,32,58-61}$ results using T34A would suggest that its role is to promote sensitivity to chemotherapy and radiotherapy. In the first study to investigate T34A's ability to enhance cancer cell chemosensitivity, transfected survivin-T34A-induced apoptosis was as effective as Taxol alone, whereas transfected survivin-T34A cells enhanced Taxol-induced apoptosis. ${ }^{11}$ Subsequent studies involving other chemotherapeutics have also revealed enhanced apoptosis in T34A-expressing cancer cells and xenograph models (Flutamide, ${ }^{59}$ Paclitaxel $^{22}$ ). Survivin-T34A combined with Adriamycin exhibited no added effect over Adriamycin alone. ${ }^{11}$ This study was followed by studies designed to characterize phosphorylated survivin $\mathrm{Thr}^{34}$ 's protective role. Following Adriamycin treatment of tumor cells, p34(cdc2) was coimmunoprecipitated with survivin. These findings suggest that $\mathrm{p} 34$ (cdc2) phosphorylates survivin on $\mathrm{Thr}^{34}$ following Adriamycin treatment thus protects the cells against the chemotherapeutic. These results were correlated with elevated MPM-2 mitotic phosphoepitope expression. ${ }^{12}$ Although survivin-T34A combined with Adriamycin exhibited no added effect over Adriamycin alone, it may still be possible to synergistically enhance Adriamycin's effect with pretreatment of survivin-T34A. The same study further showed that increased doses of Flavopiridol treatment were associated with suppression of survivin $\mathrm{Thr}^{34}$ phosphorylation.

Human glioblastoma multiforme (GBM) cells were treated with adenoviral vectors containing T34A or survivin in vivo and in vitro. No change was seen in normal cells treated with either survivin form, but radiosensitivity was exhibited in T34A-treated cells compared with survivintreated or control cells. Several other studies have confirmed similar results, suggesting that survivin only affects the radiosensitivity of tumors cells and that radioresistance can be reduced by T34A treatment. 8,32,34,61,62 Interestingly, when cancer cells were irradiated and then treated with T34A, radiosensitivity was enhanced even more. ${ }^{58}$ Enhanced radiation sensitivity may be explained by cleavage and activation of caspase-3 in T34A-treated cells. Caspase inhibitors, except those for caspase-9, have been able to reduce apoptotic phenotypes in X-irradiation of T34A-treated cells. ${ }^{32}$ Radiosensitivity may also be induced via a caspase-independent mechanism. ${ }^{58}$ DNA double strand breakage was greater in T34A-treated cells, suggesting that T34A may interfere with the cells' ability for DNA repair postirradiation. ${ }^{58}$

\section{Conclusion}

Survivin-T34A has a rich history, having first been utilized as a mimic of nonphosphorylated survivin and now as a possible cancer therapeutic. It has potential in molecular therapy because of its minimal toxicity and its ability to induce apoptosis and immune modulation while reducing angiogenesis, metastasis, and cell cycle progression in survivin-expressing cells (Figure 3). With the discovery that a T34A-conditioned medium taken from cells that overexpress and release survivin-T34A to the extracellular space is able to kill cancer cells in vitro, ${ }^{8}$ we hope to further enhance the scientific data banks and pave new paths for clinical modalities and regimens. For this to occur, however, additional testing of this agent on normal tissues, specifically testing its 'myelosuppressive effects', will have to be completed. Also, additional studies to synergistically enhance chemo- and radiotherapies, whether in combination with or through survivin-T34A pretreatment, must be accomplished. The knowledge that will make this possible includes the elucidation of survivin-T34A target cell populations, cell-surface receptors, changes that occur in the target tissue at the molecular and cellular level, the presence or development of resistance that could limit its use, the mechanism of therapeutic action, and its site of action. As survivin and p53 are important opposing regulators of cancer, further research on their interactions and signaling 


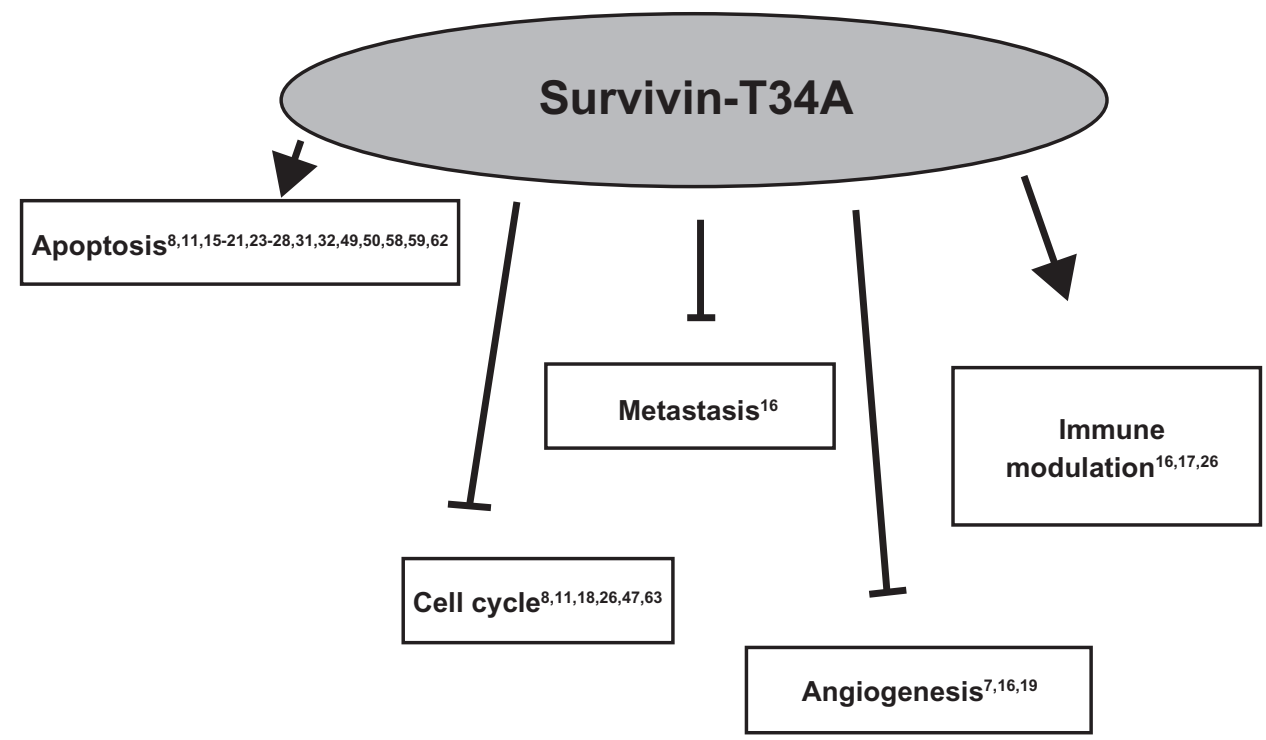

Figure 3 Survivin-T34A has therapeutic potential, as it has been shown to induce apoptosis and immune modulation while reducing angiogenesis, metastasis, and cell cycle progression in survivin-expressing cells.

pathways will likely provide specific clues for understanding this complicated relationship.

\section{Acknowledgments}

NR Wall received grant support from the NCMHD Project EXPORT Program 5P20MD001632/Project 3. We thank Dr Salma Khan, Jessica MS Jutzy, and Malyn May A Valenzuela for helpful discussion and reading of this manuscript.

\section{Disclosure}

The authors declare that they have no conflicts of interest.

\section{References}

1. Deveraux QL, Reed JC. IAP family proteins - suppressors of apoptosis. Genes Dev. 1999;13(3):239-252.

2. Hanahan D, Weinberg RA. The hallmarks of cancer. Cell. 2000;100(1): 57-70.

3. Velculescu VE, Madden SL, Zhang L, et al. Analysis of human transcriptomes. Nat Genet. 1999;23(4):387-388.

4. Reed JC. The survivin saga goes in vivo. J Clin Invest. 2001;108(7) 965-969.

5. Li F, Ambrosini G, Chu EY, et al. Control of apoptosis and mitotic spindle checkpoint by survivin. Nature. 1998;396(6711):580-584.

6. Li F. Survivin study: what is the next wave? J Cell Physiol. 2003;197(1): 8-29.

7. Andersen MH, Svane IM, Becker JC, Straten PT. The universal character of the tumor-associated antigen survivin. Clin Cancer Res. 2007; 13(20):5991-5994.

8. Khan S, Aspe JR, Asumen MG, et al. Extracellular, cell-permeable survivin inhibits apoptosis while promoting proliferative and metastatic potential. Br J Cancer. 2009;100(7):1073-1086.

9. Khan S, Jutzy JM, Aspe JR, McGregor DW, Neidigh JW, Wall NR. Survivin is released from cancer cells via exosomes. Apoptosis. 2010 Aug 18. [Epub ahead of print].

10. O'Connor DS, Grossman D, Plescia J, et al. Regulation of apoptosis at cell division by $\mathrm{p} 34 \mathrm{cdc} 2$ phosphorylation of survivin. Proc Natl Acad Sci US A. 2000;97(24):13103-13107.
11. Mesri M, Wall NR, Li J, Kim RW, Altieri DC. Cancer gene therapy using a survivin mutant adenovirus. J Clin Invest. 2001;108(7):981-990.

12. Wall NR, O’Connor DS, Plescia J, Pommier Y, Altieri DC. Suppression of survivin phosphorylation on Thr34 by flavopiridol enhances tumor cell apoptosis. Cancer Res. 2003;63(1):230-235.

13. Zhou S, Li L, Jian X, et al. The phosphorylation of survivin Thr34 by p34cdc2 in carcinogenesis of oral submucous fibrosis. Oncol Rep. 2008; 20(5):1085-1091.

14. O'Connor DS, Wall NR, Porter AC, Altieri DC. A p34(cdc2) survival checkpoint in cancer. Cancer Cell. 2002;2(1):43-54.

15. Yan H, Thomas J, Liu T, et al. Induction of melanoma cell apoptosis and inhibition of tumor growth using a cell-permeable survivin antagonist. Oncogene. 2006;25(52):6968-6974.

16. Peng XC, Yang L, Yang LP, et al. Efficient inhibition of murine breast cancer growth and metastasis by gene transferred mouse survivin Thr34->Ala mutant. J Exp Clin Cancer Res. 2008;27:46.

17. Li HX, Zhao XY, Wang L, et al. Antitumor effect of mSurvivinThr34-> Ala in murine colon carcinoma when administered intravenously. Med Oncol. 2009 Dec 1. [Epub ahead of print].

18. McKay TR, Bell S, Tenev T, et al. Procaspase 3 expression in ovarian carcinoma cells increases survivin transcription which can be countered with a dominant-negative mutant, survivin T34A; a combination gene therapy strategy. Oncogene. 2003;22(23):3539-3547.

19. Blanc-Brude OP, Mesri M, Wall NR, Plescia J, Dohi T, Altieri DC. Therapeutic targeting of the survivin pathway in cancer: initiation of mitochondrial apoptosis and suppression of tumor-associated angiogenesis. Clin Cancer Res. 2003;9(7):2683-2692.

20. Liu T, Brouha B, Grossman D. Rapid induction of mitochondrial events and caspase-independent apoptosis in survivin-targeted melanoma cells. Oncogene. 2004;23(1):39-48.

21. Wang Z, Fukuda S, Pelus LM. Survivin regulates the p53 tumor suppressor gene family. Oncogene. 2004;23(49):8146-8153.

22. Zhang M, Mukherjee N, Bermudez RS, et al. Adenovirus-mediated inhibition of survivin expression sensitizes human prostate cancer cells to paclitaxel in vitro and in vivo. Prostate. 2005;64(3):293-302.

23. Ma X, Zheng W, Wei D, et al. Construction, expression, and purification of HIV-TAT-survivin (T34A) mutant: a pro-apoptosis protein in Escherichia coli. Protein Expr Purif. 2006;47(1):36-44.

24. Shen C, Liu W, Buck AK, Reske SN. Pro-apoptosis and anti-proliferation effects of a recombinant dominant-negative survivin-T34A in human cancer cells. Anticancer Res. 2009;29(4):1423-1428. 
25. Zhang R, Wang T, Li KN, et al. A survivin double point mutant has potent inhibitory effect on the growth of hepatocellular cancer cells. Cancer Biol Ther. 2008;7(4):547-554.

26. Fukuda S, Pelus LM. Activated H-Ras regulates hematopoietic cell survival by modulating survivin. Biochem Biophys Res Commun. 2004; 323(2):636-644.

27. Yu DD, Wang CT, Shi HS, et al. Enhancement of cisplatin sensitivity in lewis lung carcinoma by liposome-mediated delivery of a survivin mutant. J Exp Clin Cancer Res. 2010;29:46.

28. Xiao W, Chen X, Yang L, Mao Y, Wei Y, Chen L. Co-delivery of doxorubicin and plasmid by a novel FGFR-mediated cationic liposome. Int J Pharm. 2010;393(1-2):119-126.

29. Grossman D, Kim PJ, Schechner JS, Altieri DC. Inhibition of melanoma tumor growth in vivo by survivin targeting. Proc Natl Acad Sci US A. 2001;98(2):635-640.

30. Dohi T, Okada K, Xia F, et al. An IAP-IAP complex inhibits apoptosis J Biol Chem. 2004;279(33):34087-34090.

31. McNeish IA, Lopes R, Bell SJ, et al. Survivin interacts with Smac/ DIABLO in ovarian carcinoma cells but is redundant in Smac-mediated apoptosis. Exp Cell Res. 2005;302(1):69-82.

32. Ogura A, Watanabe $Y$, Iizuka D, et al. Radiation-induced apoptosis of tumor cells is facilitated by inhibition of the interaction between survivin and Smac/DIABLO. Cancer Lett. 2008;259(1):71-81.

33. Boya P, Kroemer G. Lysosomal membrane permeabilization in cell death. Oncogene. 2008;27(50):6434-6451.

34. Barrett RM, Osborne TP, Wheatley SP. Phosphorylation of survivin at threonine 34 inhibits its mitotic function and enhances its cytoprotective activity. Cell Cycle. 2009;8(2):278-283.

35. Kroemer G, Pouyssegur J. Tumor cell metabolism: cancer's Achilles' heel. Cancer Cell. 2008;13(6):472-482.

36. Shin S, Sung BJ, Cho YS, et al. An anti-apoptotic protein human survivin is a direct inhibitor of caspase-3 and -7. Biochemistry. 2001;40(4): 1117-1123.

37. Haldar S, Negrini M, Monne M, Sabbioni S, Croce CM. Downregulation of bcl-2 by p53 in breast cancer cells. Cancer Res. 1994; 54(8):2095-2097.

38. Strobel T, Swanson L, Korsmeyer S, Cannistra SA. BAX enhances paclitaxel-induced apoptosis through a p53-independent pathway. Proc Natl Acad Sci U S A. 1996;93(24):14094-14099.

39. Ashcroft M, Vousden KH. Regulation of p53 stability. Oncogene. 1999; 18(53):7637-7643.

40. Marine JC, Lozano G. Mdm2-mediated ubiquitylation: p53 and beyond. Cell Death Differ. 2010;17(1):93-102.

41. Hoffman WH, Biade S, Zilfou JT, Chen J, Murphy M. Transcriptional repression of the anti-apoptotic survivin gene by wild type p53. J Biol Chem. 2002;277(5):3247-3257.

42. Irwin MS, Kaelin WG. p53 family update: p73 and p63 develop their own identities. Cell Growth Differ. 2001;12(7):337-349.

43. Yang A, Kaghad M, Caput D, McKeon F. On the shoulders of giants: p63, p73 and the rise of p53. Trends Genet. 2002;18(2):90-95.

44. Yang A, Kaghad M, Wang Y, et al. p63, a p53 homolog at 3q27-29, encodes multiple products with transactivating, death-inducing, and dominant-negative activities. Mol Cell. 1998;2(3):305-316.

45. Altieri DC. Survivin, versatile modulation of cell division and apoptosis in cancer. Oncogene. 2003;22(53):8581-8589.

OncoTargets and Therapy

\section{Publish your work in this journal}

OncoTargets and Therapy is an international, peer-reviewed, open access journal focusing on the pathological basis of all cancers, potential targets for therapy and treatment protocols employed to improve the management of cancer patients. The journal also focuses on the impact of management programs and new therapeutic agents and protocols on
46. Altieri DC. The case for survivin as a regulator of microtubule dynamics and cell-death decisions. Curr Opin Cell Biol. 2006;18(6):609-615.

47. Lens SM, Rodriguez JA, Vader G, Span SW, Giaccone G, Medema RH. Uncoupling the central spindle-associated function of the chromosomal passenger complex from its role at centromeres. Mol Biol Cell. 2006; 17(4):1897-1909.

48. Ruchaud S, Carmena M, Earnshaw WC. Chromosomal passengers: conducting cell division. Nat Rev Mol Cell Biol. 2007;8(10):798-812.

49. Shan Y, Wang C, Yang L, et al. Inhibition of human lung adenocarcinoma growth using survivint34a by low-dose systematic administration. J Biosci. 2010;35(2):209-216.

50. Pan L, Peng XC, Leng F, et al. Therapeutic effects of survivin dominant negative mutant in a mouse model of prostate cancer. J Cancer Res Clin Oncol. 2010 Mar 9. [Epub ahead of print].

51. O'Connor DS, Schechner JS, Adida C, et al. Control of apoptosis during angiogenesis by survivin expression in endothelial cells. Am J Pathol. 2000;156(2):393-398.

52. Tran J, Rak J, Sheehan C, et al. Marked induction of the IAP family antiapoptotic proteins survivin and XIAP by VEGF in vascular endothelial cells. Biochem Biophys Res Commun. 1999;264(3):781-788.

53. Harfouche R, Hassessian HM, Guo Y, et al. Mechanisms which mediate the antiapoptotic effects of angiopoietin-1 on endothelial cells. Microvasc Res. 2002;64(1):135-147.

54. Papapetropoulos A, Fulton D, Mahboubi K, et al. Angiopoietin-1 inhibits endothelial cell apoptosis via the Akt/survivin pathway. J Biol Chem. 2000; 275(13):9102-9105.

55. Mahboubi K, Li F, Plescia J, et al. Interleukin-11 up-regulates survivin expression in endothelial cells through a signal transducer and activator of transcription-3 pathway. Lab Invest. 2001;81(3):327-334.

56. Zhang M, Coen JJ, Suzuki Y, et al. Survivin is a potential mediator of prostate cancer metastasis. Int J Radiat Oncol Biol Phys. 2010;78(4): 1095-1103.

57. Melief CJ. Cancer immunotherapy by dendritic cells. Immunity. 2008; 29(3):372-383.

58. Chakravarti A, Zhai GG, Zhang M, et al. Survivin enhances radiation resistance in primary human glioblastoma cells via caspase-independent mechanisms. Oncogene. 2004;23(45):7494-7506.

59. Zhang M, Latham DE, Delaney MA, Chakravarti A. Survivin mediates resistance to antiandrogen therapy in prostate cancer. Oncogene. 2005; 24(15):2474-2482.

60. Fujie Y, Yamamoto H, Ngan CY, et al. Oxaliplatin, a potent inhibitor of survivin, enhances paclitaxel-induced apoptosis and mitotic catastrophe in colon cancer cells. Jpn J Clin Oncol. 2005;35(8):453-463.

61. Ferrario A, Rucker N, Wong S, Luna M, Gomer CJ. Survivin, a member of the inhibitor of apoptosis family, is induced by photodynamic therapy and is a target for improving treatment response. Cancer Res. 2007; 67(10):4989-4995.

62. Yuan QZ, Wang CT, Mao YQ, et al. Enhanced tumor radiosensitivity by a survivin dominant-negative mutant. Oncol Rep. 2010;23(1):97-103.

63. Canovas PM, Guadagno TM. Functional analysis of survivin in spindle assembly in Xenopus egg extracts. J Cell Biochem. 2007; 100(1):217-229.

patient perspectives such as quality of life, adherence and satisfaction The manuscript management system is completely online and includes a very quick and fair peer-review system, which is all easy to use. Visit http://www.dovepress.com/testimonials.php to read real quotes from published authors. 